\title{
Modeling of a Scheduling Method for Organizing Training Assignments
}

\author{
Ahmad Diponegoro \\ Graduate Program of Management, Postgraduate School \\ University of Muhammadiyah Prof. Dr. HAMKA \\ Jakarta, Indonesia \\ adipone@uhamka.ac.id
}

\author{
Fajar Rukman \\ Board of Research and Training \\ State Ministry of Religious Affairs \\ Jakarta, Indonesia \\ fajarrukman2809@gmail.com
}

\begin{abstract}
The objectives of this research are (i) to develop a model for managing staff assignments in an institution that organizes various events of training; and (ii) to propose a method for determining an optimal policy for the staff assignments. Two preliminary results are obtained. First, an analytical approach is employed to formulate the problem, which resulted in a multiperiod model of assignment problem. Second, a heuristic method is proposed to obtain a near-optimum solution for the problem formulated. This research is expected to contribute in providing a tool for managing operations in an educational organization.
\end{abstract}

Keywords—staff assignment; heuristic; educational operations management

\section{INTRODUCTION}

An important factor in managing an organization is prescribing efficient operation policies for the organization to survive in the current competition. This also applies in educational institutions that often organize several events of short courses or training education. In a typical educational organization's planning horizon, personnel must be assigned to staff positions in several training events. The organization must prescribe an acceptable assignment policy for personnel such that the budget can be minimized and the personnel can be fairly assigned to support their career.

Staff assignment has attracted many researchers for years. For the problem addressed here, it is formulated as a multidimensional assignment problem as stated in [1] or it can also be formulated as a multiperiod assignment problem [3] and [4]. The application of this problem category spans many areas of staff assignments, such as in medical staffing [4] and [5], in scheduling law enforcement agency to elections [6], and in assigning airline customer service agent [7].

Optimal solution to the problem of multiperiod assignment is considered computationally intractable since it is categorized as an NP-hard complexity [1] and [2]. Optimal solution methods to such a problem have been sought by several researchers, such as provided by [11], [10], [9], and [8]. Efficient heuristics are then sought by many as alternative approach for tackling large instances of the problems. Most of the heuristics are usually developed for rather specific subclass of problems. This research propose an alternative heuristic approach for a specific staff assignments problems that arise in educational organizations that hold multiple training events. Personnel of the organization are assigned to one or more staff position of a training event. Further detail of the problem addressed here will be described in the following section.

\section{PROBLEM DESCRIPTION}

Consider an organization holds several training events, or simply refer to as events for the sake of brevity. The events are scheduled in a planning horizon. In each event, a number of person are assigned to staff positions as the committee to organize it. The staffs are taken from a pool of personnel in the organization. The problem is to assign personnel into the events based on a criterion of optimality. To describe the problem, we provide the following case.

Consider $K$ number of events will be held in a planning horizon, say, a year. There are $L$ staffs position in an event for which personnel must be assigned into in order to comprise an organizing committee of the event. For the case given in the current description assume that all events are identical in the number of staffs. The relaxation of this assumption will be addressed later in this paper. The staffs are taken from a pool of $N$ personnel. Thus, in the given planning horizon there will be $M=K L$ number of staff assignments. The following situation is given: (i) each assignee can be placed in any position of the committee; (ii) each assignee receives an amount of reward; (iii) the incurred cost of an event is the sum of total reward given to the assignee; and, (iv) the budget limit of the incurred cost is relaxed. The objective is to find a set of assignments of personnel so as to minimize the total budget subject to the following constraints. Note that the total budget is the sum of costs of all events in the planning horizon.

\section{A. Constraints}

Let us state the following constraint of the problem: (a) each assignee can only be placed in exactly one position of the committee; (b) in each staff position of the committee must be placed an assignee; (c) everyone in the pool of personnel has 'almost equal' number of assignments on events in the planning horizon. It is said 'almost equal' since $M / N$, or the 
average number of assignments a person can have, may not end up in a whole number.

\section{B. Problem Formulation and Notation}

Prior to formulating the problem, we present the following notation:

$x_{i j k}=$ decision variable of person $i$ assigned on $j$-th staff position of the committee of an event $k$. The value $x_{i j}=1$ if assigned or $x_{i j}=0$ otherwise.

$a_{i j}=$ reward given to person $i$ assigned on $j$-th staff position of an event committee.

$b_{i}=$ total reward given to person $i$.

Hence, the assignment problem is formulated as follows. Find $x_{i j}$ so as to

$$
\operatorname{mininimize} Z=\sum_{k=1}^{K} \sum_{j=1}^{L} \sum_{i=1}^{N} a_{i j} x_{i j k}
$$

subject to

$$
\begin{aligned}
& \sum_{j=1}^{L} x_{i j k} \leq 1 \text { for } k=1, \ldots, K ; \text { for } i=1, \ldots, N \\
& \sum_{i=1}^{N} x_{i j k}=1 \text { for } j=1, \ldots, L \text { and for } k=1, \ldots, K \\
& \sum_{k=1}^{K} x_{i j k} \leq\lceil M / N\rceil \text { for } i=1, \ldots, N \\
& \sum_{k=1}^{K} x_{i j k} \geq\lfloor M / N\rfloor \text { for } i=1, \ldots, N \\
& x_{i j k}=\{0,1\} \forall i, j, k
\end{aligned}
$$

where

$$
x_{i j k}= \begin{cases}1 & \text { if person } i \text { assigned to } j \text { position at event } k \\ 0 & \text { otherwise }\end{cases}
$$

The first constraint (2) assures a person cannot be assigned to more than one staff position in each event. The second constraint (3) assures that each staff position in an event must be assigned a person on it, i.e., a blank staff position is not allowed. The constraints (4) and (5) assure every person has 'almost equal' number of assignments on events in the planning horizon. The last constraint (6) assures the decision variable takes binary value.

The problem presented in (1) - (6) above can be categorized as a special case to a Generalized Assignment Problem. Specifically, it is of the three-dimensional assignment problem, in which the problem is of an NP-Hard complexity. A solution to the problem using a generalized optimal approach, such as Branch and Bound can be computationally intractable. Therefore, a more efficient heruistic algorithm approach is sought in this research in order to obtain an acceptable solution within reasonable time.

\section{SOLUTION APPROACH}

In general, the algorithm proposed here, referred to as Algorithm A, comprises of two main steps, setup phase and iteration phase. We will describe the method using an illustrative example. In the setup phase, a matrix is employed to streamline the description of the iterative algorithm.

\section{Algorithm A:}

1. Setup Phase: Set up a matrix of the size $N$ rows $\times(K L+K$ +1 ) columns. Entries of the matrix are grouped by events, as shown in Fig. 1. The initial values of the $x_{i j k}$ 's are zero. Every entry in the right column at each group of event $k$ are the number of assignment of a person in the corresponding event, referred to as the subtotal column $S_{i k}$. The initial value of the entries in this column should also be 0 (zero). The final column, rereferred to as $S T_{i}$, is the sum of the subtotal $S_{i 1}, \ldots, S_{i K}$ of the corresponding row $i$.

\section{Iteration Phase:}

a. For each event $k$

i. Select person $i$ in the event $k$ that corresponds with the smallest value of $S T_{i}$ to be assigned to the position of staff (column) $j$ that has not had anyone being assigned into. If there are more than one choices of person $i$ to be assigned to choices of position $j$ to be assigned, choose the one with the corresponding smallest cost $a_{i j}$ in which the corresponding entries of the matrix are not given cross mark. Set the value of the corresponding decision variable $x_{i j k} \leftarrow 1$. Put cross marks on the entries of the other rows of the corresponding column $j$.

ii. Set the value of $S_{i k} \leftarrow 1$ and put cross marks on the entries of the other columns of staff assignments of the same row $i$ within the group event $k$. Update the value of $S T_{i}$ of the corresponding row $i$ by adding $S T_{i} \leftarrow S T_{i}+1$.

iii. If the value of $S T_{i}$ from the previous step (i.e., 2a.iii) fits the constraints (4) and (5), that is,

$$
\lfloor M / N\rfloor \leq S T_{i} \leq\lceil M / N\rceil,
$$

then cross the entries of the other columns of the same row $i$ for all staff assignments of all event $k$. Thus, the corresponding person $i$ cannot be assigned again into the remaining events. Else, go the next step.

iv. Repeat Step 2.a.i for the remaining staff position $j$ of the event $k$.

b. Repeat Step 2.a if on any remaining events $k$ there are empty staff positions. Else STOP $\square$
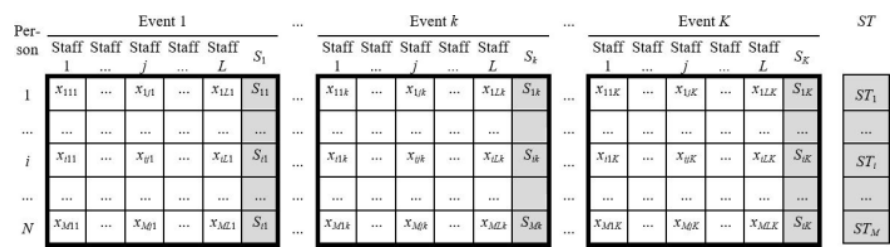

Fig. 1. Iterative Matrix 


\section{A. Computational complexity}

In the problem addressed here, there are $N$ personnel to be assigned into $M$ number of assignments. From the Algorithm $A$ described previously, it can be computed that the computational complexity is of the order $O(M \times N)$. Therefore, the problem if of polynomial complexity.

The following instance of problem will provide an illustration of the application of the algorithm described above. We keep the problem size small for brevity of the illustration.

\section{B. Solution for assignment of personnel to events}

Consider a plan for assigning 4 (four) personnel into 4 (four) events, in which for each event there are three staff positions, with the cost matrix given in Fig. 2. The dimension of the cost matrix for every event is 4 by 3 , in which there are four of them of the identical size.

The initial matrix resulted from the Setup Phase of the Algorithm $A$ is provided in Fig. 3. The dimension of the matrix is, therefore 4 by 17 . The entries of the matrix are zeros.

Here, we have $K=4, L=3, M=12, N=4$, and each person will be assigned to $M / N=3$ staff positions on 4 events. We start the first iteration process from Event 1, although it is not prevented to start from any other events. We choose to set $x_{121}$ $\leftarrow 1$ since the corresponding $a_{121}=1$ is among the lowest cost available in the corresponding event. Hence, we put cross marks on column 2 of the remaining rows within the Event 1, set $S_{1,1} \leftarrow 1$, put cross marks on the remaining columns of row 1 within Event 1, and update the value of $S T_{1} \leftarrow S T_{1}+1$. At this point $S T_{1}=1$, in which the constraints (4) and (5) are not satisfied for $i=1$. The corresponding matrix for the current state of iteration is provided on 0 as follows. The steps 2.a.i through 2.a.iv are then repeated.

Upon completing the iteration steps 2.a.i through 2.a.iv repeatedly, the assignments of all staff of Event 1 are obtained. The following matrix of Fig. 5 provides the current state.
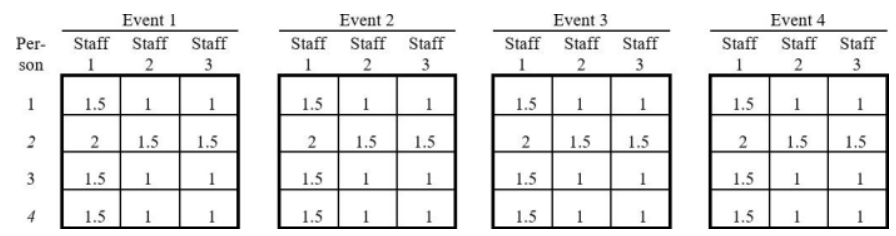

Fig. 2. Cost matrix of the the problem of the size 4 by 3 by 4 .

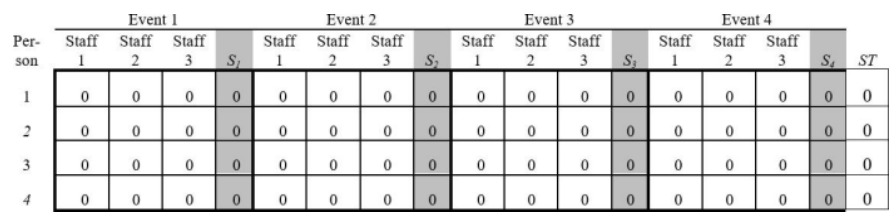

Fig. 3. Initial matrix from the Setup Phase

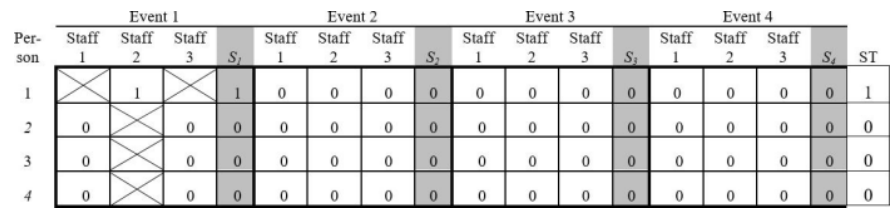

Fig. 4. The resulting matrix from the first iteration of steps 2.a.i through 2.a.iii.

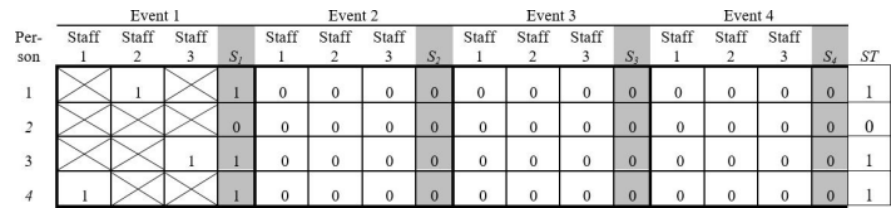

Fig. 5. The resulting matrix from assigning staffs of Event 1.

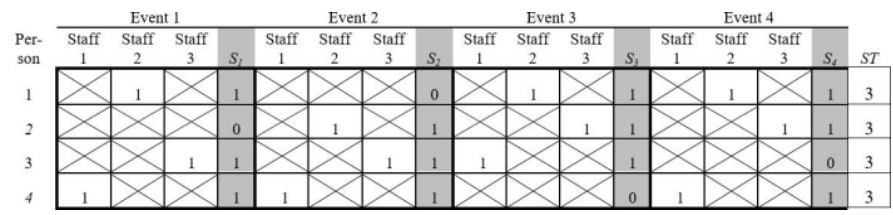

Fig. 6. Final matrix of assignments.

Going through all the iteration steps to obtain staff assignment in the given four events in planning horizon, we can obtain the result in the matrix given in Fig. 6.

The solution obtained is, therefore, $x_{1,2,1}=1, x_{3,3,1}=1, x_{4,1,1}$ $=1, x_{2,2,2}=1, x_{3,3,2}=1, x_{4,1,2}=1, x_{1,3,3}=1, x_{2,3,3}=1, x_{3,1,3}=1$, $x_{1,2,4}=1, x_{2,3,4}=1, x_{4,1,4}=1$; and, else $x_{i j k}=0$. The corresponding cost function value is $z=15.5$.

\section{DISCUSSIONS}

The proposed problem formulation implicitly assumes that the number of staff positions are identical for all events in the planning horizon. This is due to our intention to provide a rather succinct presentation of our proposed approach. Without loss of generality, however, our formulation can be applied on cases where the number of staff assignments are not necessarily identical for all events. In the latter cases, $M$ is simply equal to the sum of all number of assignments of all the events. Consequently, the cost formulation in (1) and the constraint (3) shall be adapted into

$$
Z=\sum_{k=1}^{K} \sum_{j=1}^{L_{k}} \sum_{i=1}^{N} a_{i j} x_{i j k}
$$

and

$$
\sum_{j=1}^{L_{k}} x_{i j k} \leq 1 \text { for } k=1, \ldots, K \text {; for } i=1, \ldots, N
$$

respectively. In the updated formulation given above, the size of assignment in each event is denoted by $L_{k}$, for $k=1, \ldots, K$. In the assignment matrix, the size of staff matrices follows the number of staff positions $L_{k}$ in every event in the planning horizon. The proposed solution approach goven on this research can be applied accordingly without any modification of the steps of iteration.

\section{CONCLUDING REMARKS}

In this paper a problem formulation of staff assignment on events of training in a planning horizon has been provided. An efficient solution methodology that uses heuristic approach has been proposed. The method determines the assignment of personnel into staff positions. The proposed solution method 
provides a practical approach for managers in solving typical personnel assignment. The model developed is limited to assuming a rule of distributing assignments uniformly among personnel of an organization. Further research may be directed on more general situation where various number of assignment are prescribed in an organization policy. In order to enrich the applicability of the research, a given budget constraint can be incorporated into the problem formulation.

\section{REFERENCES}

[1] K. C. Gilbert and R. B. Hofstra, "Multidimensional assignment problems," Decision Sciences, vol. 19, no. 2, pp. 306-321, 1988.

[2] M. L. Fisher, R. Jaikumar and L. N. Van Wassenhove, "A multiplier adjustment method for the generalized assignment problem," Management Science, vol. 32, no. 9, pp. 1095-1103, 1986.

[3] L. S. Franz, H. M. Baker, G. K. Leong and T. R. Rakes, "A mathematical model for scheduling and staffing multiclinic health regions," European Journal of Operational Research, vol. 41, no. 3, pp. 277-289, 1989.

[4] L. S. Franz and J. L. Miller, "Scheduling Medical Residents to Rotations: Solving the Large-Scale Multiperiod Staff Assignment Problem," Operations Research, vol. 41, no. 2, pp. 269-279, 1993.
[5] J. Guo, D. R. Morrison and S. H. Jacobson, "Complexity results for the basic residency scheduling problem," Journal of Scheduling, vol. 17, no. 3, p. 211-223, 2014.

[6] B. Nag, "A MIP model for scheduling India's General elections and police movement," OPSEARCH, vol. 51, no. 4, p. 562-576, 2014.

[7] Y.-H. Kuo, J. M. Leung and C. A. Yano, "Scheduling of multi-skilled staff across multiple locations," Production and Operations Management, vol. 23, no. 4, p. 626-644, 2014

[8] C. Vogiatzis, E. L. Pasiliao and P. M. Pardalos, "Graph partitions for the multidimensional assignment problem," Computational Optimization and Applications, vol. 58, no. 1, p. 205-224, 2014.

[9] M. Larsen, "Branch and bound solution of the multidimensional assignment problem formulation of data association," Optimization Methods and Software, vol. 27, no. 6, pp. 1101-1126, 2012.

[10] E. L. Pasiliao, "Local Neighborhoods for the Multidimensional Assignment Problem," in Dynamics of Information Systems: Theory and Applications, New York, Springer New York, 2010, pp. 353-371.

[11] A. J. Robertson, "A set of Greedy Randomized Adaptive Local Search Procedure (GRASP) implementations for the multidimensional assignment problem," Computational Optimization and Applications, vol. 19 , no. 2 , p. 145-164, 2001. 